\title{
Improving the Accuracy of the MFIE with the Choice of Basis Functions ${ }^{1}$
}

\author{
Özgür Ergül* and Levent Gürel \\ Department of Electrical and Electronics Engineering \\ Bilkent University \\ Ankara, Turkey \\ (ergul@ee.bilkent.edu.tr, lgurel@bilkent.edu.tr)
}

\section{Introduction}

In the method-of-moments (MOM) [1] and the fast-multiple-method (FMM) [2] solutions of the electromagnetic scattering problems modeled by arbitrary planar triangulations, the magnetic-field integral equation (MFIE) can be observed to give less accurate results compared to the electric-field integral equation (EFIE), if the current is expanded with the Rao-Wilton-Glisson (RWG) [3] basis functions. The inaccuracy is more evident for problem geometries with sharp edges or tips [4]. This paper shows that the accuracy of the MFIE depends strongly on the quality of the current modeling and that the accuracy can be significantly improved by the choice of the basis functions. Comparisons are performed for four different basis functions.

\section{The Use of Basis Functions in the MFIE}

Application of the MOM on the MFIE requires the evaluation of the impedance matrix elements

$$
Z_{m n}=-\int_{S_{m}} d \bar{r} \tilde{t}_{m}(\bar{r}) \cdot \hat{n} \times \int_{S_{n}} d \bar{r}^{\prime} \bar{b}_{n}\left(\bar{r}^{\prime}\right) \times \nabla^{\prime} g\left(\bar{r}, \bar{r}^{\prime}\right),
$$

where $\bar{t}_{m}$ and $\bar{b}_{n}$ represent the testing and basis functions, tespectively. Different from the interaction expression for the EFIE [3], calculation of Equation (1) does not put any restriction on the choice of the basis and testing functions. Therefore, both divergenceconforming and curl-conforming functions can be used to expand the current, if the interactions will be calculated by this equation. On the other hand, replacement of the $\nabla$ operator on the basis leads to a new expression as

$$
\begin{aligned}
Z_{m n} & =\int_{S_{m}} d \bar{r} \bar{t}_{m}(\bar{r}) \cdot \hat{n} \times \int_{S_{n}} d \bar{r}^{\prime} g\left(\bar{r}, \bar{r}^{\prime}\right) \nabla_{S}^{\prime} \times \bar{b}_{n}\left(\bar{r}^{\prime}\right) \\
& -\int_{S_{m}} d \bar{r} \bar{t}_{m}(\bar{r}) \cdot \hat{n} \times \int_{S_{n}} d \bar{r}^{\prime} \nabla_{S}^{\prime} \times\left\{g\left(\bar{r}, \bar{r}^{\prime}\right) \bar{b}_{n}\left(\bar{r}^{\prime}\right)\right\} \\
& -\int_{S_{m}} d \bar{r} \bar{t}_{m}(\bar{r}) \cdot \hat{n} \times \int_{S_{n}} d \bar{r}^{\prime} \bar{b}_{n}\left(\bar{r}^{\prime}\right) \times \hat{n}^{\prime} \frac{\partial}{\partial \mathrm{n}^{\prime}} g\left(\bar{r}, \bar{r}^{\prime}\right),
\end{aligned}
$$

I This work was supported by the Turkish Academy of Sciences in the framework of the Young Scientist Award Program (LG/TUBA-GEBIP/2002-1-12), and by the Scientific and Technical Research Council of Turkey (TUBITAK) under Research Grant 103 E008. 
where the basis is restricted to be curl-conforming. Similarly, the operator can be put on the testing function, and this time the testing function is restricted to be curl-conforming.

In order to investigate the accuracy of the MFIE, we used four different basis functions; $\mathrm{RWG}, \mathrm{TL}, \mathrm{n} \times \mathrm{RWG}$ and $\mathrm{n} \times \mathrm{TL}$ defined on the planar triangles. The TL functions are proposed in [5] to obtain better representation of the current distributions calculated by the EFIE formulation, and they are divergence-conforming similar to the RWG functions. The latter two functions are simply obtained by performing the cross product of the surface normal with the RWG and TL functions, and these are curl-conforming functions. $\mathrm{TL}$ and $\mathrm{n} \times \mathrm{TL}$ functions are more inefficient than the RWG and $\mathrm{n} \times \mathrm{RWG}$ functions in terms of the problem size, since two of these functions are located at each edge of the triangulation.

For the divergence-conforming functions, Equation (I) is used with the application of the singularity extraction for the basis integral as given in [6]. For the curl-conforming functions, Equation (2) is preferred since the second integral turns into a line integral and the singularity extraction becomes easier for the overall calculation. For all of the implementations, Galerkin method is applied and testing functions are chosen to be the same as the basis functions.

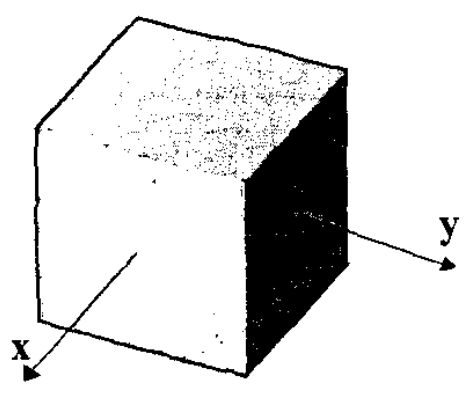

(a)

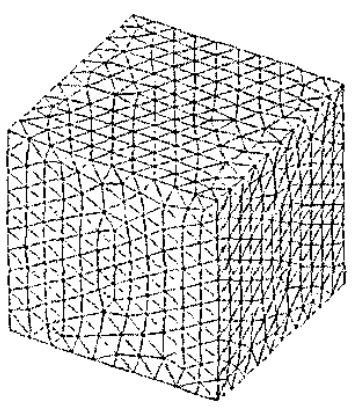

(b)

Figure 1 . (a) Cube with $1 \lambda$ edge, (b) triangulation with $\lambda 10$ mesh size.

\section{Results}

Figure 1(a) shows a scattering problem that involves a cube with $1 \lambda$ edge. The cube is triangulated with $\mathcal{N} 10$ mesh size (Figure $1(b)$ ) and an incident field propagates in the $-x$ direction with a y-polarized electric field. Figure 2 (a) represents the magnitude of the $y$ (dominant) component of the induced current on the $x=0.5$ (front) surface found by the FMM implementations using the MFIE formulation and different basis functions. It can be observed that the current is not modeled sufficiently with the RWG functions. The curl-conforming $n \times R W G$ gives better representation; but the TL and $n \times$ TL functions have even better performances. The singularity of the current near the edges can be observed to be significantly better modeled with the latter two functions. 
Figure 3 shows the total radar cross section (RCS) values on the $x-y$ plane for the cube geometry in Figure 1(a). For all types of the basis functions, the mesh size is changed from $\lambda / 5$ to $\lambda / 20$. It can be observed that the RCS curves are converging faster for the TL or $\mathrm{n} \times \mathrm{TL}$ basis functions. The RCS values obtained by the $\lambda / 10$ mesh size is more reliable for these functions as compared to the RWG function that leads to very slow convergence.

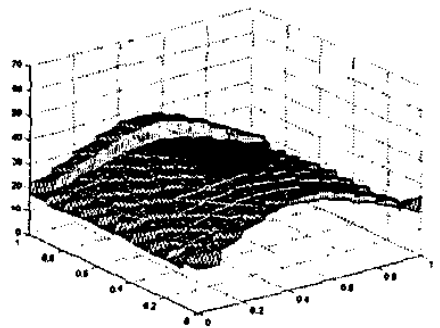

(a)

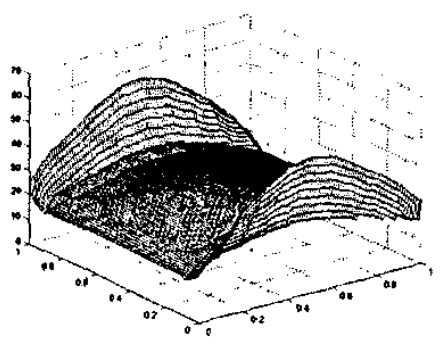

(c)

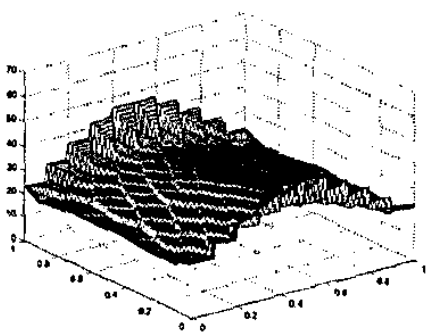

(b)

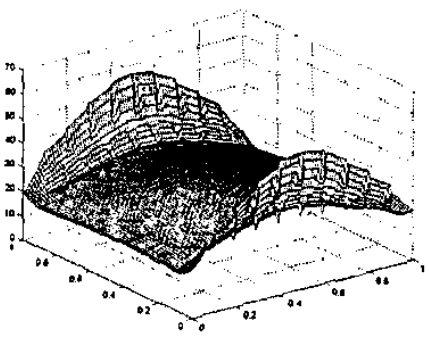

(d)

Figure 2. Magnitude of the y component of the induced current on the front surface of the cube in Figure 1(a) represented by (a) RWG, (b) $n \times R W G$, (c) TL and (d) $n \times T L$ functions.

\section{Conclusion}

The accuracy of the MFIE for the MOM and FMM solutions of the electromagnetic scattering problems modeled by arbitrary planar triangulations can be significantly improved by the choice of basis functions. The current distribution and the RCS results can be observed to be more accurate for the TL and $\mathrm{n} \times \mathrm{TL}$ functions. With the usual choice of the mesh size as $\lambda / 10$, the values obtained by the MFIE with RWG basis functions are not very reliable if the geometry of the problem has sharp edges and tips. For these problems, TL and $\mathbf{n} \times$ TL functions can be used to improve the efficiency in spite of the increased computational cost. 


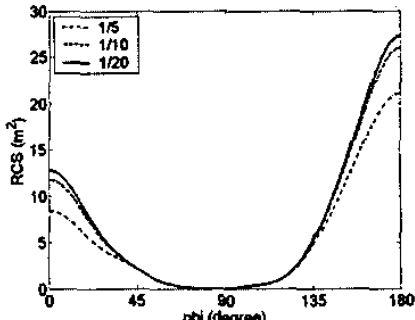

(a)

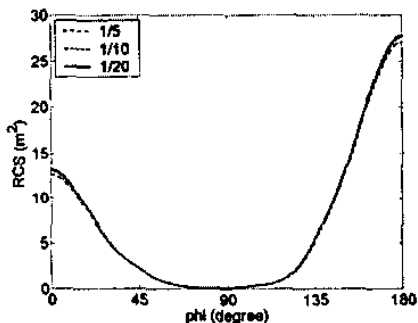

(c)

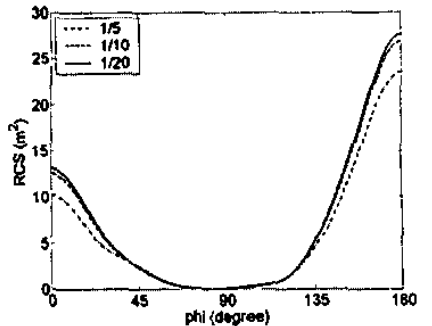

(b)

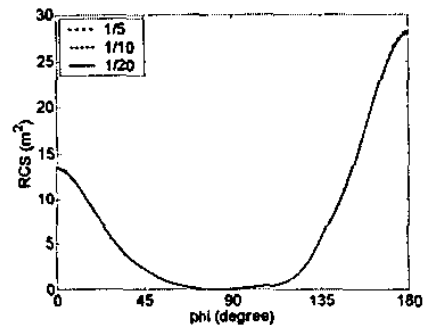

(d)

Figure 3. RCS values on the $x-y$ plane for the cube geometry with different mesh sizes obtained by the use of (a) RWG, (b) $n \times R W G$, (c) TL and (d) $n \times$ TL functions in MFIE formulation.

\section{References}

[1] R. F. Harrington, Field Computation by Moment Methods. IEEE Press, 1993.

[2] R. Coifman, V. Rokhlin, and S. Wandzura, "The fast multipole method (FMM) for the wave equation: a pedestrian prescription," IEEE Ant. Propag. Mag., vol. 35, no. 3, pp. 7-12, June 1993.

[3] S. M. Rao, D. R. Wilton, and A. W. Glisson, "Electromagnetic scattering by surfaces of arbitrary shape," IEEE Trans. Antennas Propagat., vol. AP-30, pp. 409-418, May 1982.

[4] Ö. Ergül and L. Gurrel, "Investigation of the Inaccuracy of the MFIE Discretized with the RWG Basis Functions," 2004 IEEE AP-S International Symposium and URSI Radio Science Meeting, Monterey, CA, June 2004.

[5] L. C. Trintinalia and H. Ling, "An improved triangular patch basis for the method of moments," 2000 IEEE AP-S International Symposium and URSI Radio Science Meeting, Salt Lake City, UT, vol. 4, pp. 2306-2309, July 2000.

[6] R. E. Hodges and Y, Rahmat-Samii, "The evaluation of MFIE integrals with the use of vector triangle basis functions," Micro. Opr. Tech. Lett., vol. 14, no. 1, pp. 9-14, Jan. 1997. 